\title{
Role of tumor location on high-grade serous ovarian cancer prognosis
}

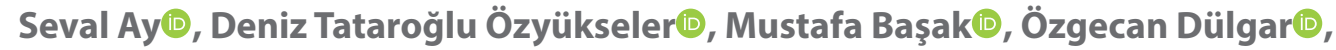

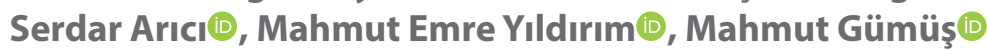 \\ Medical Oncology Clinic, Istanbul Medeniyet University, Turkey
}

\begin{abstract}
Objectives: Ovarian cancer is associated with the highest mortality of gynecologic cancers. Epidemiological and genetic factors of ovarian cancer development are clearly defined but prognostic factors have not been adequately identified. Right and left ovarian cancers seem to act different behaviors at high-grade serous ovarian cancer (HGSOC) prognosis. The aim of this study is to explain this prognostic role of its sidedness.

Material and methods: We reviewed 160 consecutive patients with Figo stage 1-3 HGSOCs and undergone surgery at two high-volume hospitals. Prognostic effects of primary tumor location onset were evaluated in terms of 5-year disease free survival and overall survival rate.

Results: One hundred-sixty patients with ovarian cancer records were analyzed using the Kaplan-Meier method, that demonstrated a significant difference in the 5 -year disease-free survival rates between right and left-sided cancers for all stages $(44.6 \%$ vs $78.5 \%, \mathrm{p}<0.001)$. Also, there was significant difference in the 5 -year overall survival rates between the two groups ( $71.1 \%$ vs $91.9 \%, p=0.020)$.

Conclusions: Tumor location within the HGSOC seems to be a compelling prognostic factor ovarian cancer. Further prospective studies are needed in order to support our hypothesis.

Key words: disease free survival; high-grade serous ovarian cancer; left and right side cancers; overall survival; prognostic values; tumor location
\end{abstract}

Ginekologia Polska 2022; 93, 4: 284-289

\section{INTRODUCTION}

Ovarian cancer is the second most common gynecologic cancer. Furthermore, it is associated with the highest mortality of gynecologic cancers in the western world. According to the results, every year, 230000 new ovarian cancer patients will be diagnosed, and 150000 of those are likely to die [1]. Due to the lack of warning symptoms and the absence of screening recommendations, approximately $70 \%$ of cases diagnosed with advanced disease [2].

Invasive ovarian cancers evaluated into three main types: epithelial ovarian cancers, sex-cord stromal tumors, and germ cell tumors. Epithelial ovarian cancers are the most common types and associated with aggressive behavior and a high relapse rate. Epithelial ovarian cancer is a heterogeneous disease with four major histologic sub- types as the serous, endometrioid, mucinous, and clear cell. High-grade serous ovarian cancer (HGSOC) is the most common (70\%) and aggressive subtype of all disease [3]. At early-stage disease, patients have a five-year survival rate of $92 \%$, although this rate decreases to $29 \%$ at advanced stage disease [4]. Also, approximately $75 \%$ of patients diagnosed at an advanced stage.

Embryonic left-right asymmetry causes different responses to external influences. At malignancies asymmetry is best described is colon cancer. Many studies reported clinical, pathological and in the molecular biological pattern differences between right- sided colon carcinomas (RCC) and left-sided colon carcinomas (LCC) [5]. The differing molecular characteristics translate into a differential clinical outcome with RCC displaying a poor prognosis [6]. Literature suggests

\footnotetext{
Corresponding author:

Seval Ay

Medical Oncology Clinic, Istanbul Medeniyet University, Turkey

phone: +905064832979, e-mail: drsevalay@gmail.com
} 
that site of the primary tumor within the colon have prognostic and treatment implications. However, some studies investigated the laterality of cutaneous melanoma and the excess of left-sided tumors seemed to appear to statistically significantly more than right sided tumors. Reason of this could be differences in sun exposure and/or asymmetry of melanocyte distribution or characteristics arising at the time of embryological development $[7,8]$.

This article determines an overview of ovarian cancer and the prognostic role of its sidedness. Recent studies showed us lymph nodes are asymmetrical in the right and left axis of human; although fewer lymph nodes on the left side, they are more hypertrophic [9]. This study aims to evaluate the effect of lateralization of tumor side on disease-free survival (DFS) and overall survival (OS) rates.

\section{MATERIAL AND METHODS Study population}

The database of the Medical Oncology Department of two high-volume hospitals was used to identify all patients diagnosed with ovarian cancer. All patients in this study underwent curative resection for ovarian cancer [10]. The histopathological staging was confirmed postoperatively by a consulting pathologist, according to The International Federation of Obstetrics and Gynecology (FIGO) staging system. Patients' data were collected from medical records and clinical follow-up visits. Demographic characteristics of patients included age at diagnosis, body mass index (BMI), marital status, number of births, educational status, menopause status, and occupation.

Two hundred thirty patients with HGSOC were investigated; after analyzing the medical records, 160 patients were chosen according to the following inclusion criteria: (1) Patients with ovarian cancer originated from one side clearly; left or right (2) diagnosed as epithelia, high grade serous ovarian cancer, (3) staged by International Federation of Gynecology and Obstetrics (FIGO) as stage 1 to 3 who underwent total hysterectomy, salpingo-oophorectomy, pelvic and para-aortic lymphadenectomy, omentectomy, and resected any suspicious or enlarged disease.

Patients meet the following criteria were excluded: (1) cancer that originated from both sides or unclear (2) patients at age under 18 (3) who had more than one solid tumor history (4) staged as stage 4 disease according to FIGO.

Parameters were evaluated as categories when through analysis, and these categories' relationships with DFS and OS were examined. DFS was calculated from the date of ovarian cancer resection to the time of proven recurrence. OS was calculated from the date of ovarian cancer resection until the time of death from any cause or the last follow up time.

All procedures performed in studies involving human participants were in accordance with the ethical stan- dards of the institutional and/or national research committee and with the 1964 Helsinki declaration and its later amendments or comparable ethical standards; was approved by the Ethics Committee of The Institutional Review Board of Istanbul Medeniyet University Hospital local Ethics Committee (IRB No. 2020/0066).

\section{Adjuvant chemotherapy and follow up}

The adjuvant treatment regimen contained carboplatin (AUC 5) and paclitaxel $\left(175 \mathrm{mg} / \mathrm{m}^{2}\right)$ on day 1 . This cycle was repeated every three weeks. The planned treatment duration, according to our standard institutional protocol was six cycles. Most patients were followed according to our institutional protocol, which consisted mainly of physical examination, measurement of the serum tumor marker [cancer antigen 125 (CA 125)], and computed tomography.

For the remaining patients, information regarding clinical outcome and survival was obtained by telephone interviews with the patients or their relatives. Tumor recurrence was detected by physical examination, serum CA 125 assay, and chest, abdominal, and pelvic imaging every 3-6 months for two years, and then every six months for the following three years [11]. After five years, patients had annual follow-up examinations unless they have an emerging complaint. We started analyzing patients with HGSOC retrospectively from December 12, 2014. The cutoff date for our analysis was July 20, 2019.

\section{Statistical methods}

The demographic and clinicopathological features of the study population were stratified according to primary ovarian tumor side. Categorical variables were expressed as count and percentage, and the differences were tested using the Chi-square test or Fisher's exact test when appropriate. DFS was calculated from the date of ovarian cancer resection to the time of proven recurrence or death. OS was calculated from the date of ovarian cancer resection until the time of death from any cause or of the latest follow-up. Five-year DFS and OS were estimated using the Kaplan-Meier method. The effect difference between factors was determined by the Log-rank test. Age, BMI, number of births, and FIGO staging was assessed as a prognostic factor for OS and DFS in Cox regression analysis. The reported $\mathrm{p}$-values were two-sided, and $p$-values $<0.05$ were considered statistically significant. All data were analyzed using SPSS statistical software Version 17.0 (SPSS Inc, Chicago, IL, USA).

\section{RESULTS \\ Patient characteristics}

We analyzed the medical records of 160 patients HGSOC. The median age was 56 years (26-84); the median age of menarche was $13(12-16)$; the median number of births 
Table 1. Clinicopathological characteristics of right- and left-sided ovarian cancer

\begin{tabular}{|c|c|c|c|c|}
\hline \multirow{2}{*}{ Characteristics } & All & Right-sided cancer & Left-sided cancer & \multirow{2}{*}{$\mathbf{p}$} \\
\hline & $(n=160)$ & $\mathrm{n}(\%)$ & $n(\%)$ & \\
\hline $\begin{array}{l}\text { Age } \\
\quad \leq 50 \\
>50\end{array}$ & $\begin{array}{c}52 \\
108\end{array}$ & $\begin{array}{l}28(32.6) \\
58(67.4)\end{array}$ & $\begin{array}{l}24(32.4) \\
50(67.6)\end{array}$ & 0.986 \\
\hline $\begin{array}{l}\text { BMI } \\
\quad<25 \\
\geq 25\end{array}$ & $\begin{array}{c}46 \\
114\end{array}$ & $\begin{array}{l}22(25.6) \\
64(74.4)\end{array}$ & $\begin{array}{l}24(32.4) \\
50(67.6)\end{array}$ & 0.340 \\
\hline $\begin{array}{l}\text { No of birth } \\
\leq 2 \\
>2\end{array}$ & $\begin{array}{l}78 \\
82\end{array}$ & $\begin{array}{l}44(51.2) \\
42(48.8)\end{array}$ & $\begin{array}{l}34(46.0) \\
40(54.0)\end{array}$ & 0.510 \\
\hline $\begin{array}{c}\text { Occupation } \\
\text { Employee } \\
\text { Housewife }\end{array}$ & $\begin{array}{c}35 \\
125\end{array}$ & $\begin{array}{l}22(25.6) \\
64(74.4)\end{array}$ & $\begin{array}{l}13(17.6) \\
61(82.4)\end{array}$ & 0.221 \\
\hline $\begin{array}{l}\text { Marital Status } \\
\text { Single } \\
\text { Married }\end{array}$ & $\begin{array}{c}47 \\
113\end{array}$ & $\begin{array}{l}29(33.7) \\
57(66.3)\end{array}$ & $\begin{array}{l}18(24.3) \\
56(75.7)\end{array}$ & 0.193 \\
\hline $\begin{array}{l}\text { FIGO } \\
\text { Stage 1-2 } \\
\text { Stage } 3\end{array}$ & $\begin{array}{l}88 \\
72\end{array}$ & $\begin{array}{l}35(40.7) \\
51(59.3)\end{array}$ & $\begin{array}{l}53 \text { (71.6.) } \\
21(28.4)\end{array}$ & $<0.001$ \\
\hline $\begin{array}{l}\text { Menopause status } \\
\text { Postmenopausal } \\
\text { Premenopausal }\end{array}$ & $\begin{array}{c}105 \\
55\end{array}$ & $\begin{array}{l}60(69.8) \\
26(30.2)\end{array}$ & $\begin{array}{l}45(60.8) \\
29(39.2)\end{array}$ & 0.234 \\
\hline $\begin{array}{l}\text { Lymph node } \\
\text { Positive } \\
\text { Negative }\end{array}$ & $\begin{array}{c}31 \\
129\end{array}$ & $\begin{array}{l}24(27.9) \\
62(72.1)\end{array}$ & $\begin{array}{c}7(9.5) \\
67(90.5)\end{array}$ & 0.003 \\
\hline
\end{tabular}

$\mathrm{BMI}$ - body mass index; $\mathrm{FIGO}$ - the International Federation of Obstetrics and Gynecology

was $3(0-11)$. Right-sided ovarian cancer was present in $53.8 \%(86 / 160)$ of the included patients. The clinical and pathologic characteristics of the right-sided and left-sided ovarian cancer patients are summarized in Table 1. According to patients' characteristics, at right sided group $74.4 \%$ and $67.6 \%$ left sided group BMI were $\geq 25$. In addition, $66.3 \%$ of right sided tumor patients and $75.4 \%$ of left sided tumor patients were married; $69.8 \%$ of right sided tumor patients and $60.8 \%$ of left sided tumor patients were postmenopausal. There was no statistical significance in the distribution of these characteristic features. The patients were homogeneously distributed.

A higher percentage of patients with right-sided cancers had FIGO stage 3 compared with left-sided cancer patients (59.3\% vs $28.4 \%, p<0.001)$. The percentages of the lymph node involvement in right-sided and left-sided ovarian cancer patients showed significant difference ( $27.9 \%$ vs $9.5 \%$; $p=0.003)$.

\section{Adjuvant chemotherapy}

Among the 160 patients, 147 patients received adjuvant chemotherapy. In right-sided ovarian cancers, 79 (91.9\%) patients received adjuvant chemotherapy, and in the left-sided ovarian cancers, 68 (91.9\%) patients received adjuvant chemotherapy. There was no significant difference in treatment

\begin{tabular}{|c|c|c|c|}
\hline Items & $\begin{array}{c}\text { Right-sided } \\
\text { cancer }(n=86)\end{array}$ & $\begin{array}{c}\text { Left-sided } \\
\text { cancer }(n=74)\end{array}$ & $p$ \\
\hline Treatment model & & & 0.994 \\
\hline Surgery alone & $7(8.1)$ & $6(8.1)$ & \\
\hline Adjuvant CT & $79(91.9)$ & $68(91.9)$ & \\
\hline
\end{tabular}

CT - chemotherapy

regimens between right- and left-sided ovarian cancers. Adjuvant chemotherapy is listed in Table 2.

\section{Survival analysis by tumor location}

The Kaplan-Meier survival curves demonstrated a significant difference in the 5 -year DFS rates between right and left-sided cancers for all stages $(44.6 \%$ vs $78.5 \%, p<0.001$ ) (Fig. 1). Also, there was a significant difference in the 5 -year OS rates between the two groups (71.1\% vs $91.9 \%$, $\mathrm{p}=0.020)$ (Fig. 2).

\section{Details of recurrence}

Postoperative recurrence occurred in 56 patients, 41 of whom had right-sided ovarian cancer, and 15 of whom had left-sided ovarian cancer ( $47.7 \%$ vs $20.3 \%$, p < 0.001 ). 


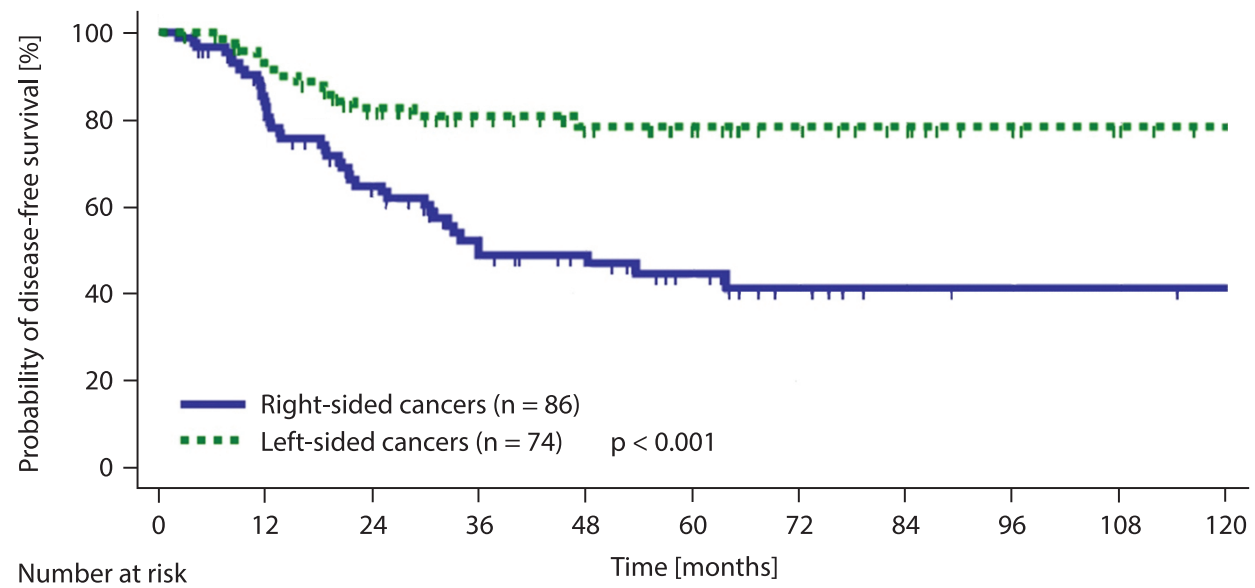

Group: Right-sided cancers $(n=86)$

$\begin{array}{lllllllllll}86 & 67 & 46 & 29 & 24 & 16 & 8 & 3 & 2 & 2 & 1\end{array}$

Group: Left-sided cancers $(n=74) \quad p<0.001$

$\begin{array}{lllllllllll}74 & 65 & 51 & 40 & 33 & 27 & 21 & 17 & 11 & 8 & 6\end{array}$

Figure 1. Five-year disease-free survival rate of left sided over cancer and right sided over cancer at diagnosis

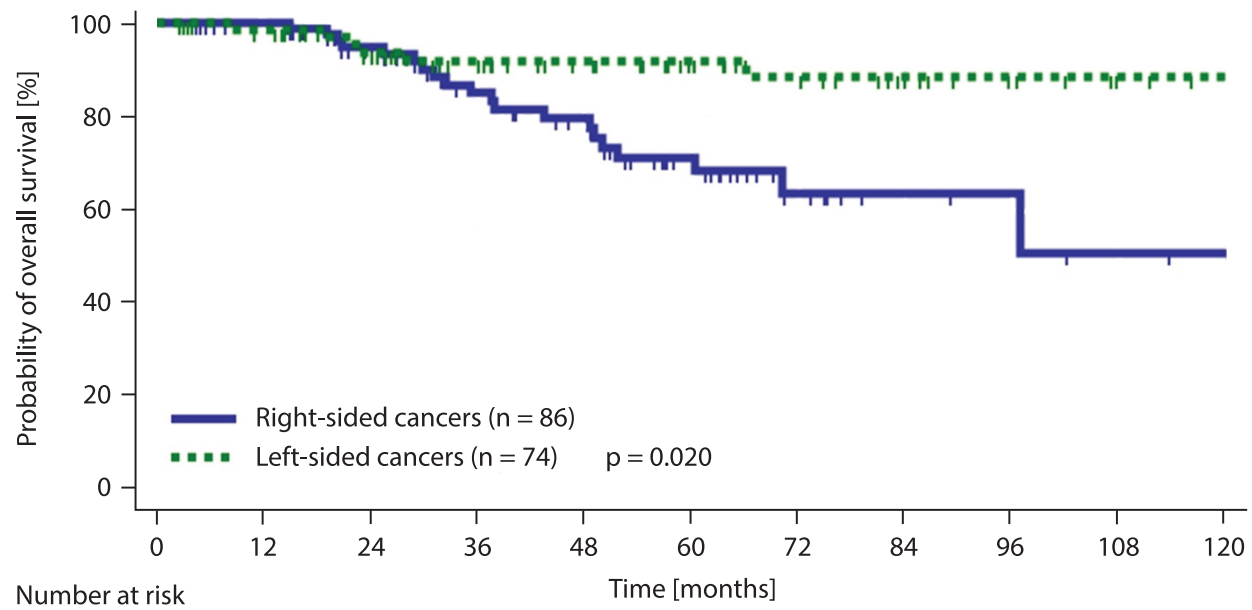

Group: Right-sided cancers $(n=86)$

$\begin{array}{ccccccccccc}86 & 81 & 66 & 50 & 39 & 25 & 12 & 6 & 5 & 3 & 2 \\ \text { Group: Left-sided cancers }(\mathrm{n}=74) & \mathrm{p}=0.020 & & & & & & & \\ 74 & 68 & 57 & 47 & 39 & 31 & 25 & 18 & 13 & 9 & 7\end{array}$

Figure 2. Five-year overall survival rate of left sided over cancer and right sided over cancer at diagnosis

\section{Multivariate analysis}

Several variables were significant predictors of outcomes in the multivariate survival models (Tab. 3 and 4). According to multivariate analyses, the FIGO staging of patients makes a significant difference in DFS and OS.

\section{DISCUSSION}

In the present study, we enrolled 160 patients with HGSOC ovarian carcinoma, and we divided into two groups originating from the right or left ovary. It was significant that right-sided ovarian cancers are worse disease-free survival and overall survival in five years than left-sided ovarian cancers. Numbers of right and left-sided ovarian tumors were homogenous and chosen from two centers consecutively.

Increased survival rate at left-sided ovarian cancers than right-sided ovarian cancers was studied before by Roychoudhuri et al. [12]. This study contains not only ovarian cancer lateralization also compares five major paired organs of the body. They investigated five-year survival rates and left-sided ovarian cancer results were significantly higher than the right-sided disease, but the difference was not significant. In this study, both ovarian epithelial 


\begin{tabular}{|c|c|c|}
\hline \multirow{2}{*}{ Variables } & \multicolumn{2}{|c|}{ 5-year DFS } \\
\hline & HR (95\% Cl) & $p$ value \\
\hline $\operatorname{Age}(\leq 50,>50)$ & $2.0(0.8-5.0)$ & 0.134 \\
\hline Menopausal status & $0.6(0.2-1.4)$ & 0.206 \\
\hline Tumor location (right, left) & $0.6(0.3-1.1)$ & 0.05 \\
\hline FIGO $(1-2,3)$ & $6.2(3.1-12.5)$ & $<0.001$ \\
\hline
\end{tabular}

$\mathrm{HR}$ - hazard ratio; $\mathrm{Cl}$ - confidence interval; DFS - disease-free survival; $\mathrm{FIGO}$ - the International Federation of Obstetrics and Gynecology

Table 4. Multivariate adjusted Cox model for 5-year overall survival

\begin{tabular}{|l|c|c|}
\multirow{2}{*}{ Variables } & \multicolumn{2}{|c|}{ 5-year OS } \\
\cline { 2 - 3 } & HR (95\% Cl) & p value \\
\hline Age $(\leq 50,>50)$ & $0.9(0.6-1.4)$ & 0.641 \\
\hline $\operatorname{BMI}(<25, \geq 25)$ & $0.6(0.4-0.9)$ & 0.007 \\
\hline Menopausal status & $2.1(1.3-3.4)$ & 0.004 \\
\hline Tumor localization (right, left) & $0.7(0.5-0.9)$ & 0.033 \\
\hline
\end{tabular}

$\mathrm{HR}$ - hazard ratio; $\mathrm{Cl}$ — confidence interval; OS — overall survival; BMI — body mass index

and germ-cell cancers were included, and the excess of right-sided ovarian germ-cell cancers was evident in most age-groups; this could have masked the significance. In addition, stage 4 disease were included to patient population. In our study all patients are chosen from $\mathrm{HGSOC}$ and we excluded metastatic disease.

Except for ovarian cancers, the presence of metastases in the contralateral organ at first diagnosis is infrequent. Reason for late presentation ovarian cancers occur after metastasis to the contralateral ovary; both ovaries are mostly involved during the presentation. This hypothesis thought to be the reason for obscuring the original primary laterality.

Right-sided lateralization of ovarian cancers was consistent with a study performed by Dane et al. [13]. This trial included 221 women diagnosed with ovarian cancer; 130 on the right side and 91 on the left side who went under systematic pelvic laparotomy lymphadenectomy. The numbers of lymph nodes were significantly higher on the right side than the ones on the left side. The reason for this could be from partial functional immune asymmetry [14]. Our study's findings, which are consistent with those data of the literature, show that there is an asymmetry of ovarian cancer behaviors that are more aggressive on the right side.

Asymmetry of delayed-type hypersensitivity reaction was reviewed in the left and right paws of mice by Gontova et al. [15]. The immune reaction was more manifested in the left paw rather than the right paw of all mice and concluded that it is determined by the functional asymmetry of regional lymph nodes. This result is verified by Erdem et al. [16]. The tuberculin skin test is applied both left and right forearms of participants previously sensitized by the BCG vaccine. The reaction was greater on the left side of the body. The stronger cell-mediated immune activity on the left side may be associated with the blocking of the metastatic invasion of cancer cells and better disease-free survival and overall survival rates at left-sided ovarian cancer. This theory could explain better survival results of left ovarian cancers than right ovarian cancers and consistent with our findings.

David H. Brewster et al., (2007) and The Surveillance, Epidemiology, and End Results (SEER) Program at 2005 [8] have observed left-sided cutaneous melanomas are more often in several different populations. One of the theories to explain laterisation was excess of left-sided tumors might arise from differential migration of melanocytes from the neural crest in the embryo. Asymmetric immune reactions are mentioned above this article. Melanoma reaction could be more powerful to exposure of sunlight at left side and end up on excess of left sided melanomas [7, 8].

There is strong evidence that that tumor subsite location differences at colon cancers are associated with prognosis and treatment implications and an independent risk factor for mortality. Wray et al. [17], analyzed 87,586 cases; $48 \%$ had tumors located in the proximal colon, whereas $10 \%$ had transverse colon cancer, $42 \%$ rectosigmoid colon. In this study, left-sided colon cancers were observed to have lower tumor grade and independently decreased mortality compared with right-sided tumors. This data was verified by Benedix et al. [18], at 2011; 53\% had RCC, and $47 \%$ had LCC followed for five years. Tumors of the RCC displayed more aggressive tumor growth patterns than LCC. This trial showed that colonic subsite provides additional prognostic information. In our study, we demonstrated the prognostic importance of tumor localization of ovarian cancers the same as colon cancer survival dataset. Similar to colon cancer our study showed us ovarian cancers should be evaluated differently from which side tumor arises. DFS in five years was significantly longer at left-sided ovarian cancers, and additionally, overall survival was shorter at right-sided ovarian cancers.

In the cancer of testicles which are embryogenic equivalents of ovaries, had same asymmetry. Stone J.M. et al. [19], found $54 \%$ of testicular tumors overall were right-sided. Seminomas, yolk sac tumors and teratomas were more right-sided malignancies than average $(p=0.02)$. They explained reason of this asymmetry might be left testis usually hangs lower than right testis, thus more susceptible to trauma. Roychoudhuri R et al. [12], found that testicular can- 
cers were more often in the right side when compared with the left side. In addition, left sided testis cancer had better OS than right sided tumor statistical significantly $(p<0.05)$. Lateralization that is noted at testicular cancers are similar to our findings of ovarian cancer. We found DFS and OS were significantly shorter at right-sided ovarian cancers. Embryogenic cell distributions or molecular feature could cause this asymmetry. Either immunity at left side might be stronger than right side, that blocks carcinogenesis.

Our study has several limitations; patients were collected from two hospitals, and surgical operations were performed by different surgeons. But operations were optimal, and no residual disease was seen. The number of one-sided onset ovarian cancer was less than we expected. Reason of this is the primary tumor could be difficult to define as right or left ovary in the first diagnosis due to the metastasis to the opposite ovary. Despite the limited number of ovarian cancers in our study, it was statistically significant that lateralization seems to be important in disease-free survival and overall survival rates in five years.

\section{CONCLUSIONS}

In summary, tumor location within the ovarian cancer is a prognostic factor for stage-3 HGSOC. This situation raises this question: 'Should we perform more aggressive surgical approaches at right-sided HGSOC?' and/or' Right sided HGSOC needed more often follow up and prolonged treatments?'. This finding could be useful for stratifying patients to determine treatment strategies at diagnosis if supported by further prospective studies.

\section{Acknowledgements}

The authors thank all participants and colleagues for their invaluable contribution to the study. This research did not receive any specific grant from agencies in the public, commercial, or not-for-profit sectors.

\section{Conflict of interest}

The authors declare no conflicts of interest.

\section{REFERENCES}

1. Ferlay J, Soerjomataram I, Dikshit R, et al. Cancer incidence and mortality worldwide: sources, methods and major patterns in GLOBOCAN 2012. Int J Cancer. 2015; 136(5): E359-E386, doi: 10.1002/ijc.29210, indexed in Pubmed: 25220842.
2. National Institutes of Health. National Cancer Institute. Surveillance, Epidemiology, and End Results Program. Statistical summaries: cancer stat fact sheets (ovary) and cancer statistics review (CSR), 1975-2013.

3. PP, Campbell K, Mishell D. Jr. Relative frequency of primary ovarian neoplasms: a 10-year review. Obstet Gynecol. 1989; 74: 926-921.

4. Reid BM, Permuth JB, Sellers TA. Epidemiology of ovarian cancer: a review. Cancer Biol Med. 2017; 14(1): 9-32, doi: 10.20892/j.issn.20953941.2016.0084, indexed in Pubmed: 28443200.

5. Bilimoria KY, Palis B, Stewart AK, et al. Impact of tumor location on nodal evaluation for colon cancer. Dis Colon Rectum. 2008; 51(2): 154-161, doi: 10.1007/s10350-007-9114-2, indexed in Pubmed: 18172729.

6. Guinney J, Dienstmann R, Wang $X$, et al. The consensus molecular subtypes of colorectal cancer. Nat Med. 2015; 21(11): 1350-1356, doi: 10.1038/nm.3967, indexed in Pubmed: 26457759.

7. Brewster DH, Horner MJD, Rowan S, et al. Left-sided excess of invasive cutaneous melanoma in six countries. Eur J Cancer. 2007; 43(18): 2634-2637, doi: 10.1016/j.ejca.2007.09.021, indexed in Pubmed: 17988856.

8. Surveillance, Epidemiology, and End Results (SEER) Program. SEER*Stat Database: Incidence - SEER 17 Regs Limited-Use, November 2005 Sub (1973-2003 varying) - Linked to county attributes - Total US, 1969-2003 Counties, National Cancer Institute, DCCPS, Surveillance Research Program, Cancer Statistics Branch, released April 2006, based on the November 2005 submission. www.seer.cancer.gov.

9. CAPPELLO F, BELLAFIORE M, PALMA A, et al. Study of axillary lymph node asymmetry in a female population. Journal of Anatomy. 2001; 199(5): 617-620, doi: 10.1017/s002187820100841x.

10. Hoskins WJ. Surgical staging and cytoreductive surgery of epithelial ovarian cancer. Cancer. 1993; 71(4 Suppl): 1534-1540, doi: 10.1002/cncr.2820710420, indexed in Pubmed: 8431891.

11. Esselen KM, Cronin AM, Bixel K, et al. Use of CA-125 Tests and Computed Tomographic Scans for Surveillance in Ovarian Cancer. JAMA Oncol. 2016; 2(11): 1427-1433, doi: 10.1001/jamaoncol.2016.1842, indexed in Pubmed: 27442965.

12. Roychoudhuri R, Putcha V, Møller H. Cancer and laterality: a study of the five major paired organs (UK). Cancer Causes Control. 2006; 17(5): 655 -662, doi: 10.1007/s10552-005-0615-9, indexed in Pubmed: 16633912.

13. Dane S, Borekci B, Kadanali S. Right-sided lateralisation of ovarian cancer and right bias asymmetry for involved pelvic lymph nodes by ovarian cancer cells. Laterality. 2008; 13(5): 393-402, doi: 10.1080/13576500801957636, indexed in Pubmed: 18608852.

14. DANE E. CELL-MEDIATED IMMUNE HYPERSENSITIVITY IS STRONGER IN THE LEFT SIDE OF THE BODY THAN THE RIGHT IN HEALTHY YOUNG SUBJECTS. Perceptual and Motor Skills. 2001; 93(6): 329, doi: 10.2466/pms.93.6.329-332.

15. Gontova IA, Abramov VV, Kozlov VA. Asymmetry of delayed type hypersensitivity reaction in mice. Bull Exp Biol Med. 2003; 135(1): 67-69, doi: 10.1023/a:1023454114499, indexed in Pubmed: 12717517.

16. Erdem T, Dane S, Kadi M. Cell-mediated immune hypersensitivity is stronger on non-involved side than involved side in patients with herpes zoster. International Journal of Neuroscience. 2003; 113: 1086-1081.

17. Wray C, Ziogas A, Hinojosa M, et al. Tumor Subsite Location Within the Colon Is Prognostic for Survival After Colon Cancer Diagnosis. Diseases of the Colon \& Rectum. 2009; 52(8): 1359-1366, doi: 10.1007/dcr.0b013e3181a7b7de.

18. Benedix F, Schmidt U, Mroczkowski P, et al. Study Group"Colon/Rectum Carcinoma (Primary Tumor)". Colon carcinoma--classification into right and left sided cancer or according to colonic subsite?--Analysis of 29,568 patients. Eur J Surg Oncol. 2011; 37(2): 134-139, doi: 10.1016/j. ejso.2010.12.004, indexed in Pubmed: 21193285.

19. Stone JM, Cruickshank DG, Sandeman TF, et al. Laterality, maldescent, trauma and other clinical factors in the epidemiology of testis cancer in Victoria, Australia. Br J Cancer. 1991; 64(1): 132-138, doi: 10.1038/bjc.1991.256, indexed in Pubmed: 1677257. 